\title{
Estimation of Muscular Fatigue under Electromyostimulation Using CWT
}

\author{
M. Yochum, T. Bakir, R. Lepers, and S. Binczak, Member, IEEE
}

\begin{abstract}
The aims of this study are to investigate muscular fatigue and to propose a new fatigue index based on the continuous wavelet transform (CWT) which is compared to the standard fatigue indexes from literature. Fatigue indexes are all based on the electrical activity of muscles (electromyogram) acquired during an electrically stimulated contraction thanks to two modules (electromyostimulation + electromyography recording) that can analyze EMG signals in real time during electromyostimulation. The extracted parameters are compared with each other and their sensitivity to noise is studied. The effect of truncation of M waves is then investigated, enlightening the robustness of the index obtained using CWT.
\end{abstract}

\section{Index Terms}

Electromyogram, Electromyostimulation, Muscle fatigue, truncation, wavelet.

\section{INTRODUCTION}

Electromyostimulation (ES) is of interest for muscular rehabilitation systems [1], in particular for people who have had nerve traumas like paraplegics or hemiplegics [2] and for people who have had a temporary immobilization of one or more limbs leading up to muscle atrophies. The main goal of ES is to increase the muscle mass by artificial contractions of muscles. In this way, muscles react as if during real exercise and it creates more muscular tissues [3]. Researchers are trying to characterize muscle behavior during contraction. These analyses allow them to obtain useful information which can lead to diagnose muscle diseases $[4,5]$. This method is specially adapted for paralyzed patients as acquired signal analysis could replace usual muscular feeling which is not accessible [6]. The most common device to extract muscular data is the electromyogram (EMG) [7], those data are called $\mathrm{V}_{\mathrm{EMG}}$. It represents electrical muscular activity of muscular fibers around electrodes. These electrical activities can be acquired during voluntary contractions or during ES. During an electrical evoked contraction, a typical waveform of $\mathrm{V}_{\mathrm{EMG}}$ appears between two stimulation pulses, which corresponds to $\mathrm{M}$ waves. The variations of this electrical activity lead to characterize the muscle states over a contraction, such as the muscular fatigue. From these $\mathrm{M}$ waves, some indexes are already widely used to estimate the muscular fatigue during contractions. It is the case for the peak to peak (PTP), the root mean square (RMS), the mean $\left(\mathrm{F}_{\text {mean }}\right)$ and the median frequency $\left(\mathrm{F}_{\text {med }}\right)$ [8-10]. These indexes are sensitive to noise and truncation [11], a phenomenon which appears in case of high frequency stimulation or when the muscle fatigue becomes large enough during moderate frequency stimulation. Furthermore, the previous fatigue indexes are only dependent of one aspect of $\mathrm{M}$ waves (amplitude or frequency) and do not take in account the total waveform of $\mathrm{M}$ waves. Besides, it is proved that $\mathrm{M}$ wave shape changes during ES [12]: Due to muscular fatigue, it temporally elongates and shrinks in amplitude [13]. Therefore, there changes may be used to determine the muscular fatigue [14].

The purpose of this paper is to analyze a new fatigue index based on CWT, named $\mathrm{I}_{\mathrm{CWT}}$, which focuses on the dilation of $\mathrm{M}$ wave over the stimulation. This index is compared with other fatigue indexes. Their sensitivities to noise are studied and compared to each other. This study includes also the influence of truncation. Besides, there is a correlation between the frequency parameters of EMGs and the conduction velocities of muscular fibers [15]. The same comment could be made between the amplitude of EMG and the number of muscular motor units used during a contraction [16]. Therefore, a comparative study between $\mathrm{I}_{\mathrm{CWT}}$ and the amplitude or frequency based indexes is performed enlightening a possible correlation between $\mathrm{I}_{\mathrm{CWT}}$ and some physiological characteristics of muscular fibers.

\section{Fatigue Index BASED on Wavelet AnAlysis}

During the ES, the EMG amplifier board records both the muscular electrical activity (M waves) but also the artifact of stimulation (see Fig 1.a), which needs to be removed in order to avoid interferences in the following treatments. The elimination of these artifacts is not simple because the two sources are in the same range of frequency. The method used here to remove the artifacts is based on a two-stage peak detection algorithm [17], which consists in a threshold technique. The stimulation artifacts have amplitudes higher than $\mathrm{M}$ waves, so that thresholds can be set between the maximum voltage of the signal and the maximum of $\mathrm{M}$ waves. Then, the elimination is obtained thanks to the sign of the signal derivative when a threshold is crossed. An example of a signal from which artifacts have been removed is shown in Fig. 1.b. In order to use the CWT

M. Yochum T. Bakir and S. Binczak are with the Laboratory LE2I CNRS UMR 6306, University of Burgundy, 9 avenue Alain Savary, BP47870 21078 Dijon cedex, France. (e-mail: stbinc@u-bourgogne.fr)

R. Lepers: is with the Laboratory INSERM U887, University of Burgundy, 21078 Dijon cedex, France. (e-mail: romuald.lepers@u-bourgogne.fr) 

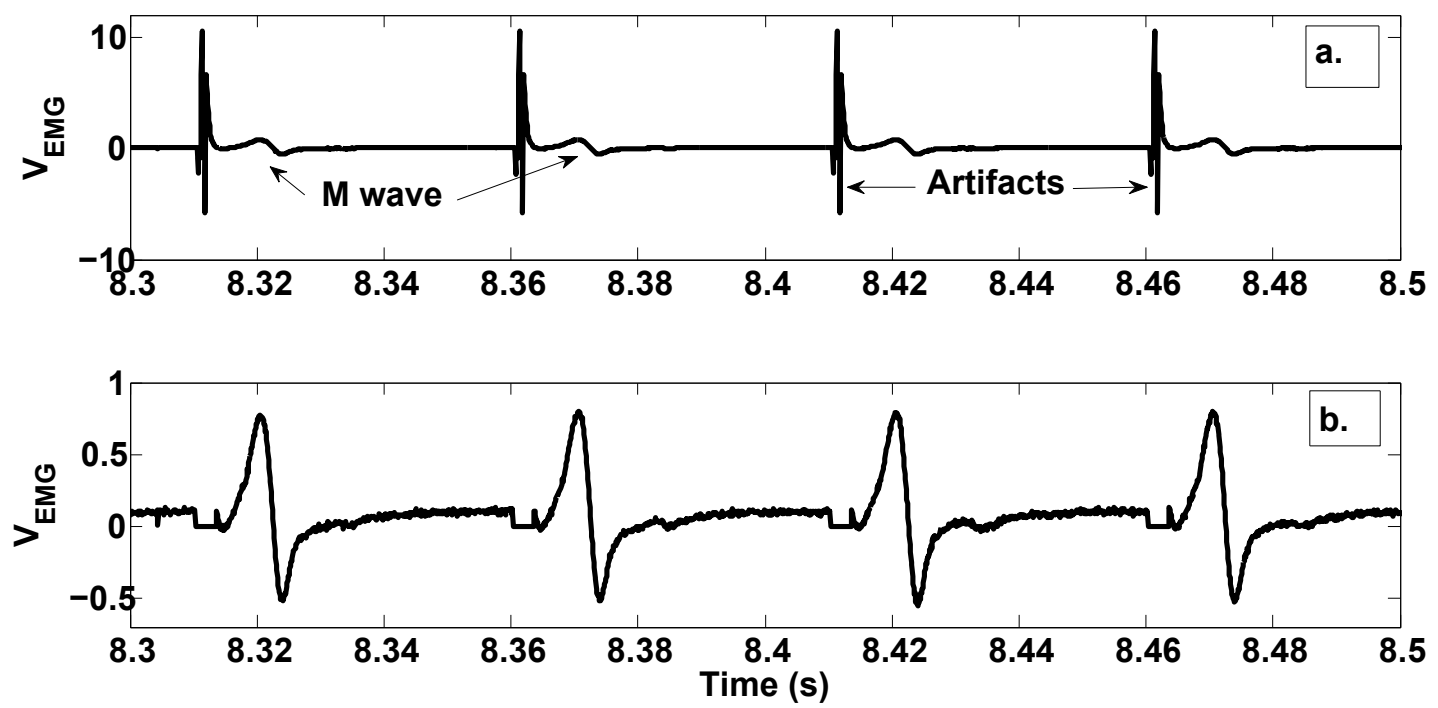

Fig. 1. Example of artifact removal. a) EMG signal from EMG board; b) EMG without artifacts.

algorithm, it is necessary to construct a wavelet which should be similar to $\mathrm{M}$ wave signal and which will act as a reference wavelet. This new wavelet is produced for each stimulation session with one of $\mathrm{M}$ waves. The principle is to use the first $\mathrm{M}$ wave of the experimentally acquired $\mathrm{M}$ waves to create an admissible wavelet. The estimated wavelet, obtained using mean least squares optimization method applied on selected $\mathrm{M}$ wave, must also have a zero mean value [18] and therefore, the eligibility condition follows

$$
A_{\hat{\psi}}=\int_{\mathbb{R}} \hat{\psi}(t) d t=0
$$

where $\hat{\psi}(t)$ is the estimated wavelet. Fig. 2 shows that the $\mathbf{M}$ wave wavelet (dashed line) matches the corresponding $\mathbf{M}$ wave (continuous line). Then, this M wave wavelet is used with the CWT. This leads to determine the correlation which exists between any $\mathrm{M}$ wave and this wavelet reference according to a temporal dilation given by

$$
C_{a, b}\left(V_{E M G}, \hat{\psi}(t)\right)=\int_{\mathbb{R}} V_{E M G} \frac{1}{\sqrt{a}} \hat{\psi}\left(\frac{t-b}{a}\right) d t,
$$

where $a$ is a scale factor (temporal dilation) and $b$ indicates the temporal location. Then, a local maximum algorithm is applied to find the highest CWT results value for each received M wave during the stimulation, as illustrated in Fig. 3. Each detected local maxima corresponds to a value of scale factor $a$ of the considered $\mathrm{M}$ wave and to a corresponding temporal location $b$. On this figure, this detection is represented by the projection on scale $a$ axis with the black arrow. Finally, the scales from local maxima are used to construct a fatigue index. The evolution of scale factor $a$ indicates an expansion undergone by the reference wavelet between the beginning $\mathrm{M}$ wave and the following. However, the common fatigue indexes usually decrease toward zero in time, contrary to CWT indexes which increase from 1 . In order to keep the same tendency, the inverse of scale parameters is taken. Therefore, the temporal dilation $I_{C W T}$ can be expressed as

$$
I_{C W T}=\left[\underset{a}{\operatorname{argmax}}\left\{C_{a, b}(s(t), \hat{\psi}(t))\right\}\right]^{-1} .
$$

Several other indexes have been implemented in order to compare them with $\mathrm{I}_{\mathrm{CWT}}$. Those treatments are directly inspired from the literature $[8-10,13,12,19]$, and include measures in amplitude of the $\mathrm{M}$ waves as Peak To Peak (PTP) and Root Mean Square (RMS) and also mean Frequency $\left(F_{\text {mean }}\right)$ and Median Frequency $\left(F_{m e d}\right)$ which are obtained by frequency based analysis. These fatigue indexes are performed for each $\mathrm{M}$ wave which are $\mathrm{V}_{\mathrm{EMG}}$ between two stimulation pulses. They are computed such as:

- PTP

$$
P T P=\max \left(V_{E M G}\right)-\min \left(V_{E M G}\right)
$$

- RMS

$$
R M S=\sqrt{\frac{1}{n} \sum_{i=0}^{n} V_{E M G}(i)^{2}}
$$




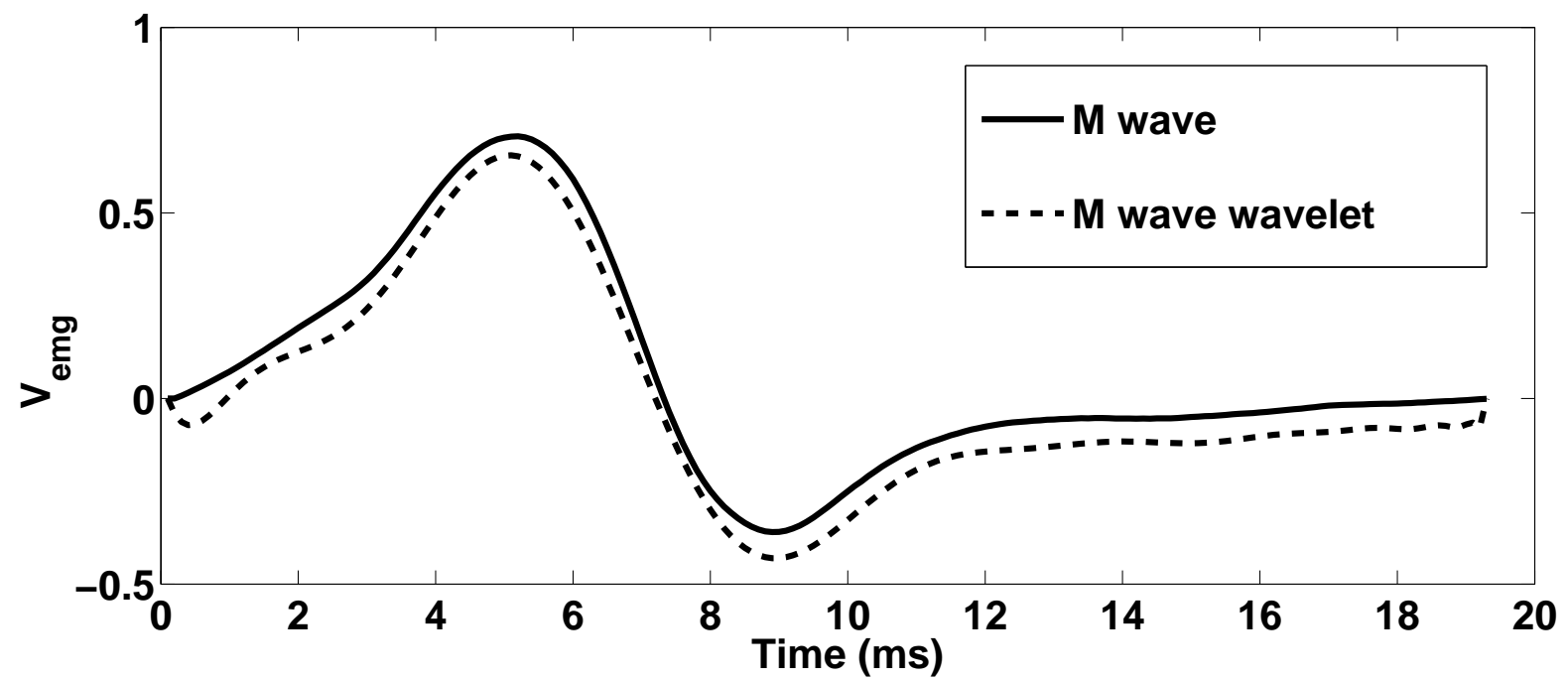

Fig. 2. M wave wavelet in dashed line compared to $M$ wave (continuous line).

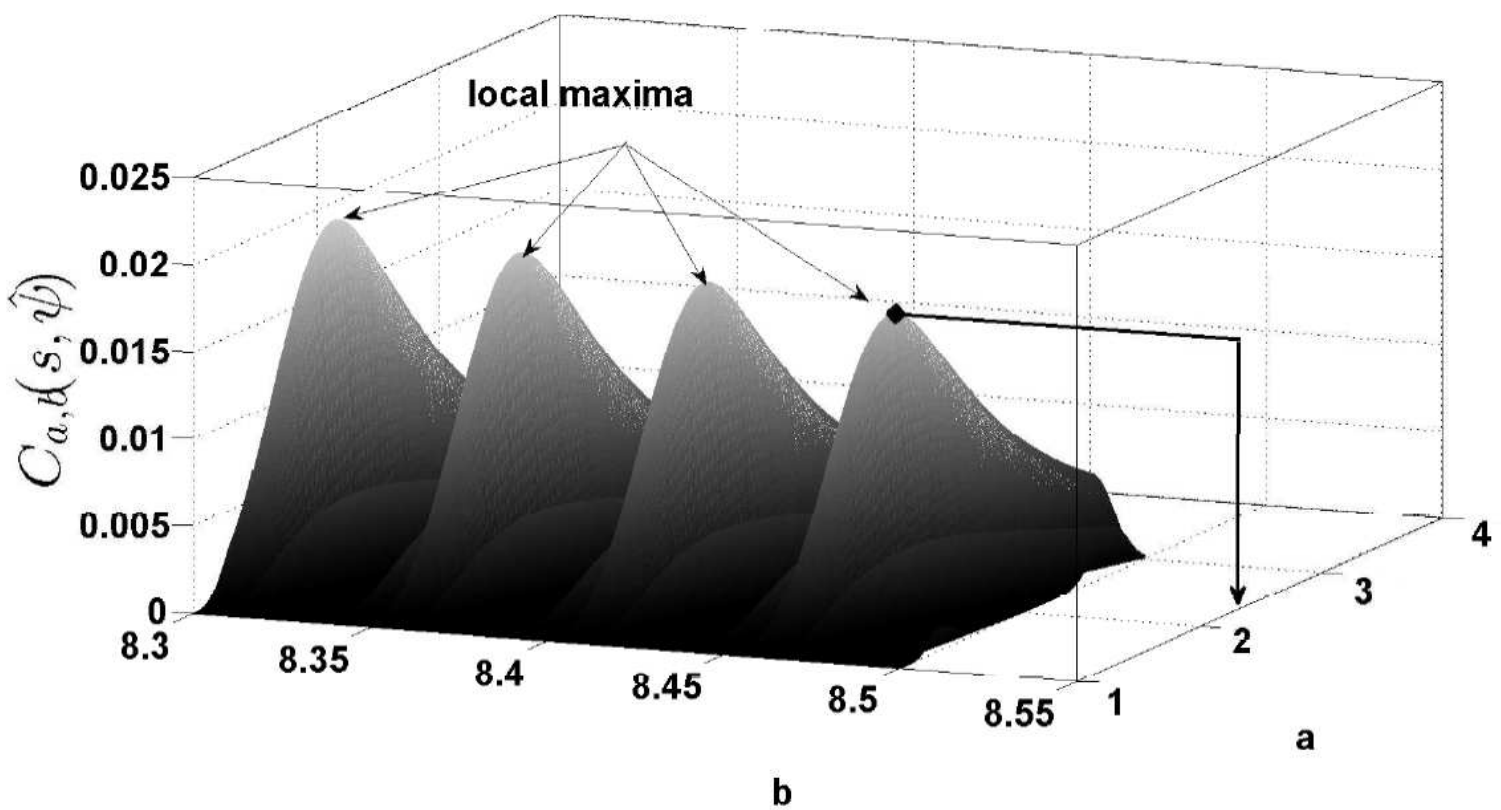

Fig. 3. $3 D$ representation of local maxima with an example of fatigue index (black arrow).

- Mean frequency

$$
F_{\text {mean }}=\frac{\sum_{i=0}^{n} P S D(i) \cdot f(i)}{\sum_{i=0}^{n} P S D(i)},
$$

where $P S D$ is the power spectrum density of $\mathrm{V}_{\mathrm{EMG}}$ and $f$ is the frequency vector.

- Median frequency

$$
\sum_{i=0}^{F_{m e d}} P S D(i)=\sum_{i=F_{m e d}}^{n} P S D(i)=\frac{1}{2} \cdot T S D,
$$

where $T S D$ is the total spectrum density. 

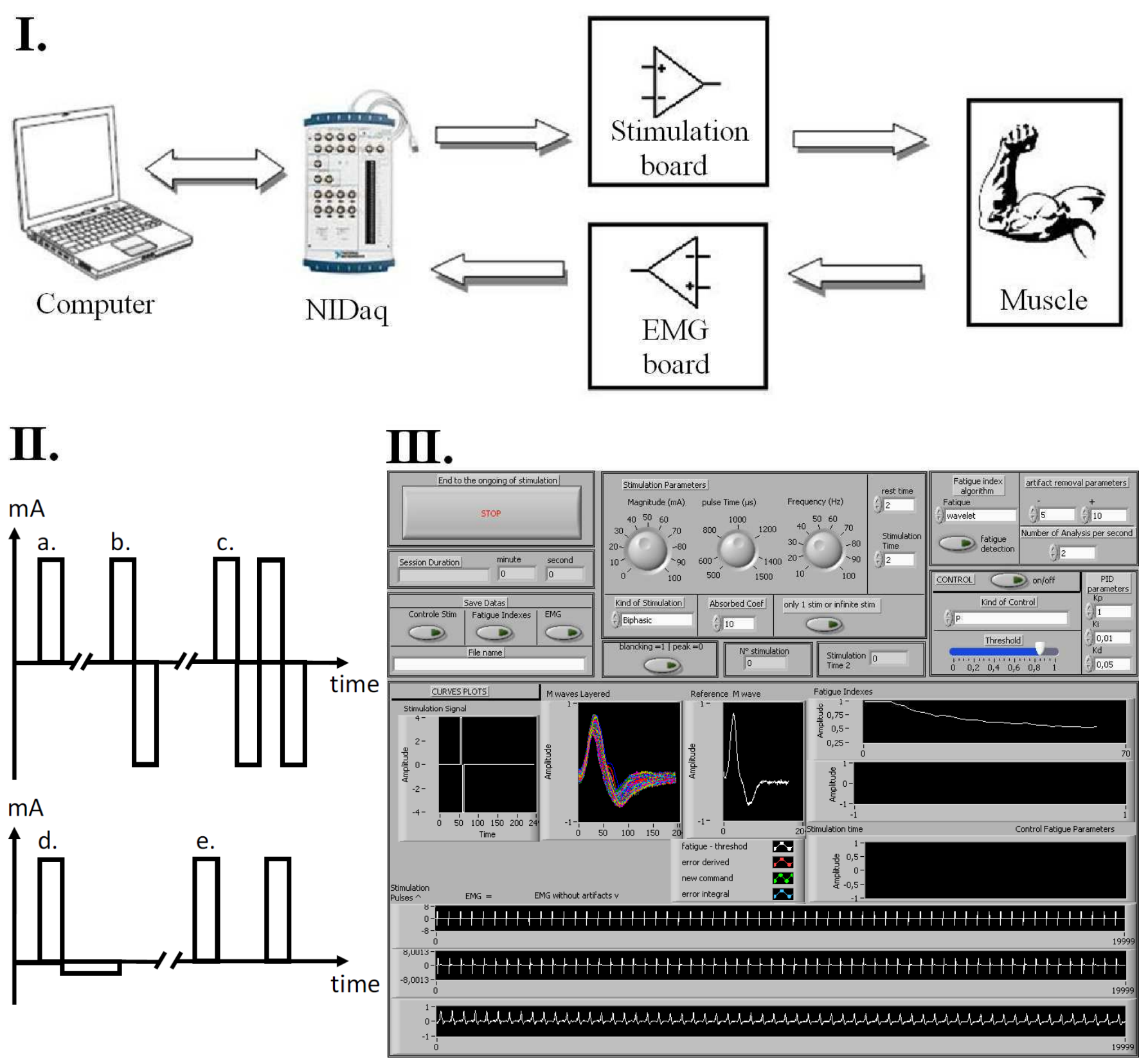

Fig. 4. I: System diagram. II: Waveforms of pulses that can be sent to the muscles. a. Monophasic. b. Biphasic. c. Dual Biphasic. d. Absorbed Biphasic. e. doublet Nlet. III: Software interface

\section{EXPERIMENTAL RESULTS}

\section{A. Experimental Setup}

We introduce an electro stimulator dedicated to the ES of a muscle and to the fatigue analysis based on EMGs feedback in real-time [20]. The Fig. 4.I presents the general diagram of the system. The device is composed of a dedicated hardware part conceived to deliver current impulse stimulations and EMGs acquisition. A software part enables the control of the stimulation and computes the fatigue indexes. A NIDaq module (NI USB-6251 BNC) from National Instruments connects these two parts in order to obtain a system processing in real-time, the myostimulation and EMG being performed at the same time.

1) Stimulation board: Each muscle has its own characteristics such as impedance, therefore, in order to obtain an accurate stimulation, the same current has to be injected in a generic way whatever the type of stimulated muscle. This task is performed by the circuit proposed by Han-Chang Wu \& al [21]. The software generates stimulation voltage pulses from $-10 \mathrm{~V}$ to $10 \mathrm{~V}$ which are converted into current pulses from $-100 \mathrm{~mA}$ to $100 \mathrm{~mA}$. In order to have controlled and precise pulses for a large range of muscular impedance, this current is maintained thanks to a symmetrical high voltage available on the board and a Wilson current mirror. 

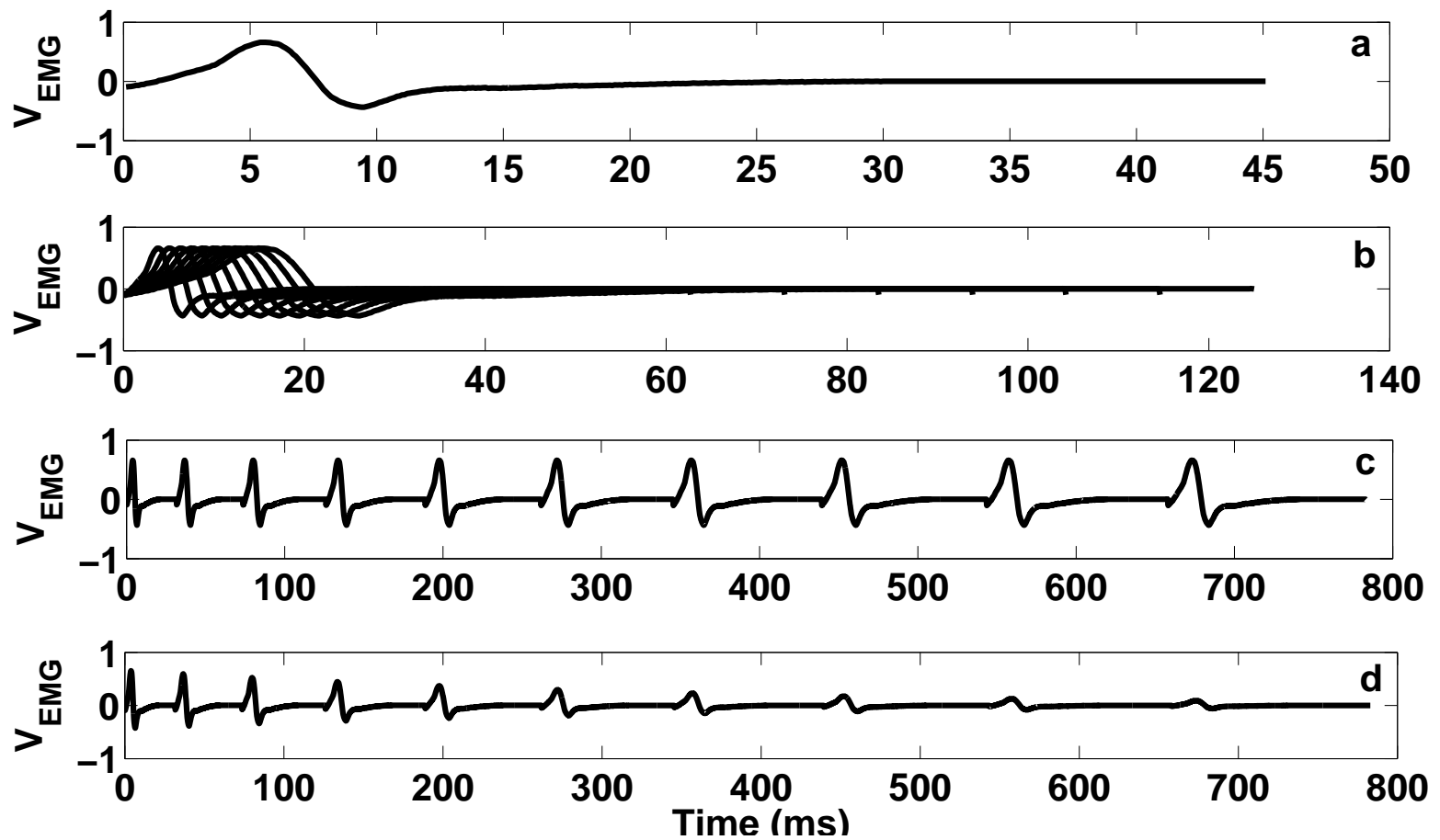

Fig. 5. a) Experimental $\mathrm{M}$ wave; b) $\mathrm{M}$ wave extended in time with a factor from 1 to 3 ; c) Artificial EMG signal with experimental extended concatenated M waves; d) lost in amplitude by an decreasing exponential to give final artificial EMG signal.

2) EMG board: Surface electrodes are used to acquire the electrical activity of the stimulated muscle. The equipment is non invasive but their positions must be accurate as EMG signals vary according to their location on the skin [22]. The setup of EMG amplifier is based on the instrumental amplifiers INA2128 from Texas Instruments [23]. This component has a 120 $\mathrm{dB}$ of Common Mode Rejection Ratio (CMRR) for a good removal of common voltage of bipolar electrodes. Three EMG electrodes are required: two for the recording of the muscular electrical activity (named $E_{1}$ and $E_{2}$ ) and another one laid on a bony point $\left(E_{r e f}\right)$ acting as a reference voltage. This board performs the difference between two electrode voltages of the muscle with a large amplification and reference erasing. The $\mathrm{V}_{\mathrm{EMG}}$ of the muscle is obtained by the difference between $E_{1}$ and $E_{2}$ such as:

$$
V_{E M G}=\left(E_{1}-E_{2}\right) \cdot G, \text { with } G=\frac{50}{R P},
$$

where $G$ is the gain of amplifier and $R P$ is an adjustable potentiometer in $k \Omega$.

3) Software: Many stimulation parameters can be adjusted though HMI (human machine interface). The current amplitude can vary from $0 \mathrm{~mA}$ to $\pm 100 \mathrm{~mA}$. As the stimulation board can supply a constant current, this one is not dependent on stimulated muscles. The duration of pulses varies from $500 \mu \mathrm{s}$ to $2000 \mu \mathrm{s}$. The range of frequency of pulse train starts at $10 \mathrm{~Hz}$ and ends at $100 \mathrm{~Hz}$. The shapes of pulses used for myostimulation can be chosen between the most common ones, such as Monophasic, Biphasic, Dual Biphasic, Asymmetric Biphasic and Doublet Nlet [24, 25] ones, but it can also be arbitrary. They are printed on Fig. 4.II. The stimulation and rest time can be adjusted, whereas EMGs and results of fatigue treatments can be saved during the stimulation process. The kind of fatigue treatment among those cited in section II is also adjustable. Values of the different parameters of the stimulation can be modified during the myostimulation. Some graphical windows are included to the HMI yielding the possibility to observe in real time the evolution of the stimulation and EMG signal parameters over time. The layered $\mathrm{M}$ wave is depicted to view the changes of the shape of $\mathrm{M}$ wave in time in comparison with reference M wave. The results of fatigue analysis selected by the user can also be represented. The Software interface is shown in the Fig. 4.III.

\section{B. Test with synthetic experimental based EMG signal}

The validation of the system is checked by using synthetic $M$ waves. The construction of this signal is based on experimental M waves acquired during ES. ES was performed on the biceps brachia with a biphasic shape, a $60 \mathrm{~mA}$ intensity, a $1 \mathrm{~ms}$ pulse duration and $30 \mathrm{~Hz}, 40 \mathrm{~Hz}, 50 \mathrm{~Hz}$ and $60 \mathrm{~Hz}$ pulse train frequencies. Five different $\mathrm{M}$ waves have been taken for each frequency. An example is shown in Fig. 5.a. Then, those standard $M$ waves are extended artificially in time, which mimics the 